\title{
Length-weight relationships of three fish species from a tropical mangrove estuary of Sarawak, Malaysia
}

\begin{abstract}
Length-weight relationships for three mangrove fish species, Setipinna melanochir (Bleeker, 1849), Ilisha elongata (Anonymous [Bennett], 1830), and Nibea soldado (Lacépède, 1802) were determined. Samplings were conducted in a tropical mangrove estuary, Sarawak, Malaysia $\left(3^{\circ} 59^{\prime} 25.76^{\prime \prime} \mathrm{N} ; 113^{\circ} 43^{\prime} 51.6^{\prime \prime} \mathrm{E}\right)$ at the peak of three different seasons using gillnets $(100 \times 2.5 \mathrm{~m}$, mesh sizes 1,2 and 4 inches) during 2014-2015. No information regarding length-weight relationships of these species was reported previously in FishBase.
\end{abstract}

Keyword: Fish species; Setipinna melanochir; Ilisha elongate; Nibea soldado; Tropical mangrove; Sarawak; Malaysia 